\title{
Psicología positiva y psicología popular de la autoayuda: un romance histórico, psicológico y cultural
}

\author{
Edgar Cabanas y Juan A. Huertas
}

Universidad Autónoma de Madrid (España)

\begin{abstract}
Resumen: En este trabajo analizamos las semejanzas y las diferencias que existen entre el discurso sobre el sujeto de la psicología positiva y el de la literatura de autoayuda. Para delimitar nuestro objeto de estudio definimos un conjunto de categorías y subcategorías psicológicas, al cual hemos denominado individualismo "positivo" y cuyo análisis cultural e histórico hemos desarrollado en otros trabajos. Además, comparamos otros factores como el tipo de vocabulario y el tipo de retórica que utilizan uno y otro tipo de literatura para defender sus argumentos. Para llevar a cabo estos análisis utilizamos una metodología de tipo mixto, en la cual combinamos el uso de la lexicometría, el ANOVA, el análisis de correspondencias y la recuperación de palabras en contexto, todo ello combinado con una discusión crítica de los textos apoyados en la bibliografía existente. El conjunto de hipótesis planteadas en este trabajo postulan que la psicología positiva y la literatura de autoayuda comparten unas raíces psicológicas comunes: persiguen el mismo horizonte de bienestar, utilizan conceptos y caracterizaciones psicológicas muy parecidas para hablar, describir y explicar al individuo, y desarrollan técnicas y prácticas similares con el fin de que los sujetos actúen sobre sí mismos en busca de su propia felicidad. También planteamos que esta comunalidad, sin embargo, no es compartida por otros tipos de psicología de objetivos prácticos e interventivos similares, como la psicoterapia cognitivo-conductual, tipo de literatura que también añadimos a los análisis. Los resultados obtenidos apuntan en la dirección del conjunto de hipótesis planteadas.

Palabras clave: psicología positiva; autoayuda; lexicometría; individualismo
\end{abstract} "positivo"; psicología crítica; felicidad; cultura norteamericana.

\section{Introducción}

En un conjunto de entrevistas que estamos realizando a consumidores de literatura disponible en secciones de "Autoayuda" y de "Psicología" en la "Casa de Libro" y en el "Fnac" de Madrid, preguntamos a "C", una estudiante de periodismo de 30 años que había comprado varios libros de autoayuda, por qué estaba interesada en este tipo de literatura y cómo utilizaba la información que obtenía de los mismos. Nos respondió que la utilizaba "porque creo que es una herramienta para...para conocerme y porque estoy pasando un momento malo...una depresión”. Añadió que estaba recibiendo una terapia en psicología positiva y que esos libros la resumían y le ayudaban a repasarla: “...es que lo que tratamos en la terapia se ve en el libro”, nos decía. Cuando le preguntamos en qué sentido los libros le ayudaban con la terapia, para nuestra sorpresa, nos contestó que el mismo autor de los libros de autoayuda era su terapeuta y por ello resumían y reflejaban tan bien lo que se decía en la consulta. Según la entrevistada no había ninguna diferencia sustancial entre las explicaciones y los consejos que le propiciaba su terapeuta y lo que el mismo terapeuta divulgaba en

* Dirección para correspondencia [Correspondence address]:

Edgar Cabanas. E-mail: edgar.cabanas@uam.es; Juan Antonio Huertas. E-mail: juanantonio.huertas@uam.es
Title: Positive psychology and self-help popular psychology: a historical, psychological and cultural romance

Abstract: In this paper, the psychological discourse about the subject provided both by positive psychology and by self-help literature is compared. To specify the object of analysis we define a set of psychological categories and subcategories, to which we have dubbed as "positive" individualism and which cultural and historical back-ground has been analyzed in previous works. Besides these categories, we analyze other aspects such as the kind of vocabulary and the type of rhetoric deployed by both kind of literature to defend their arguments. To carry out these analyses we used a mixed methodology, combining the word-count technique, the ANOVA, the correspondences analysis, and key-words in context. We added a critic discussion of the texts to the data obtained. The hypothesis set out here postulate that both positive psychology and self-help literature share common psychological roots: they defend the same idea of happiness in a similar way psychological concepts in order to describe and explain the individual, and develop analogous psychological practices and techniques aiming to help the individual to search his own well-being. We also postulate that this commonality is not shared by other psychological models, although they have a comparable interventional and practical vein, such as cognitivebehavioral psychotherapy, which we also analyze in this paper. The results obtained are congruent with the set of hypothesis here suggested.

Key words: positive psychology; self-help; word-count; "positive" individualism; critical psychology; happiness; north american culture. libros colocados en las grandes superficies bajo la etiqueta de "autoayuda".

Cuáles son las diferencias entre los consejos, las prácticas y las técnicas propuestas por la Psicología Positiva y por la psicología popular de la autoayuda - de ahora en adelante nos referiremos a ellas como "PP" y "AA", respectivamente- es una cuestión que un creciente número de psicólogos, sociólogos e historiadores críticos con la PP tratan de contestar desde que la misma apareciera en la academia. Todos estos autores coinciden en señalar que la frontera que separa la PP de la AA es principalmente institucional, no epistemológica, defendiendo que ambos tipos de literatura compartirían conceptualizaciones sobre el sujeto muy parecidas, tendrían raíces históricas y culturales similares -principalmente norteamericanas - , y responderían a intereses e ideologías comunes (Christopher, 1999; Rimke, 1997, 2000; Christopher y Hickinbottom, 2008; Miller, 2008; Norem, 2011; Binkley, 2011; Cabanas, 2011a, 2011b; Cabanas y Sánchez, 2012; Pérez-Álvarez, 2012). Así, es ampliamente aceptado que tanto la PP y la AA defienden un mismo tipo de psicología popular que, como señala Ehrenreich (2009, pp.11-12), es "ubicua y virtualmente irrevocable en la cultura americana", y que en las últimas décadas, ha sido promovida en algunos de los "talk shows" más vistos del país, como el de Larry King y el Oprah Winfrey; ha sido el material principal de best-sellers tales como el libro publicado en el 2006, El Secreto; ha sido adoptado como la teología de los más exitosos 
oradores evangélicos americanos; ha encontrado un lugar en la medicina como potencial adyuvante del tratamiento de casi cualquier enfermedad. Ha penetrado incluso en la academia en forma de "psicología positiva"... Y su alcance crece de forma global, primero en los países angloparlantes, y pronto en economías crecientes como China, Corea del Sur y la India.

Acorde con estas críticas, este trabajo defiende la tesis de que la frontera psicológica entre la PP y la AA es, cuanto menos, porosa. En el presente trabajo pondremos a prueba la hipótesis principal de que la estrecha relación existente entre ambas se debe, especialmente, a que comparten un mismo modelo psicológico de individuo, esto es, utilizan conceptos y caracterizaciones psicológicas muy parecidas para hablar, describir y explicar el comportamiento de los individuos -sus pensamientos, emociones, capacidades, actitudes, aspiraciones, motivaciones, expectativas, etc. A este modelo psicológico particular lo denominamos individualismo "positivo" (para un análisis histórico, político y económico sobre la aparición y la consolidación cultural de este modelo psicológico, ver Cabanas y Sánchez, 2012).

Como veremos a lo largo del trabajo, tal modelo está constituido por una forma peculiar, distintiva $y$ en conjunto de conceptualizar las categorías psicológicas de autocontrol, autoconocimiento, autodeterminación y autocultivo, así como de concebir las mismas como inherentemente relacionadas con una idea individualista y universalista de felicidad. Así, aunque terminológicamente estas categorías pueden ser -y de hecho soncompartidas por otras escuelas o corrientes psicológicas, tanto la PP como la AA tienen una forma particular y distintiva de conceptualizarlas, así como una preferencia por relacionarlas -al contrario que otras escuelas o corrientes psicológicas- con aspectos tales como la felicidad, la autenticidad, el florecimiento personal, la salud, el rendimiento escolar y profesional, etc.

Para poner a prueba esta hipótesis principal, dividiremos ésta en tres hipótesis secundarias o subsidiarias: 1) las categorías psicológicas que definen el individualismo "positivo" son fuertemente características tanto de la PP como de la $\mathrm{AA} ; 2)$ tales categorías, además, son igualmente características de ambos tipos de literatura; 3) estas mismas categorías son mucho menos relevantes para otras corrientes psicológicas académicas, tales como la cognitivo-conductual - de ahora en adelante, "PCC"-, la cual, si bien tiene propósitos aplicados -terapéuticos, prácticos, transformativos, etc.similares tanto a la PP como a la AA, tiene también raíces históricas, epistemológicas, filosóficas y psicológicas muy diferentes a las mismas, y, por tanto, formas diferentes de entender, explicar y dirigir el comportamiento de los individuos.

Empíricamente, el análisis de estas hipótesis podría abordarse desde niveles y métodos diferentes. Por ejemplo, desde un punto de vista cualitativo, podría adoptarse la metodología típica del "análisis crítico del discurso" (Íñiguez, 2006) o de la "psicología discursiva" (Wetherell, 1998) con el fin de estudiar aquellos "repertorios interpretativos" o "ca- tegorías discursivas" que son centrales en el tipo de caracterización y de explicación sobre el individuo que se derivan de estos tipos de literatura; o bien, desde un punto de vista más cuantitativo, podría adoptarse la metodología característica del "análisis estadístico de textos" (Lebart, Salem y Bécue, 2000), como la "lexicometría" (Baccalá, de la Cruz y Scheuer, 2002) y el "análisis factorial de correspondencias" (AFC) (Greenacre, 2008; Castellví, 1978). El uso complementario de ambos tipos de análisis, sin embargo, incrementa enormemente la validez de los resultados, y cada vez es mayor el interés en las ciencias sociales por la triangulación metodológica en la discusión de hipótesis (Leech y Onwuegbuzie, 2008; Ryan y Bernard, 2003) a través de las denominadas metodologías "mixtas" (Johnson y Onwuegbuzie, 2007).

Este trabajo utiliza este tipo de metodología, conjugando el análisis cuantitativo de la lexicometría, el ANOVA y el análisis factorial de correspondencias, con el más cualitativo del análisis contextual y el comentario de textos. Esta conjugación de metodologías nos permite analizar simultáneamente la terminología de una gran cantidad de textos temáticamente similares entre sí, contabilizar sus palabras y expresiones más frecuentes, establecer relaciones estadísticas entre determinadas "categorías psicológicas" elaboradas a partir de la construcción de diccionarios y estudiar la similitud y la diferencia entre distintos textos en función de esas mismas categorías (para otros análisis utilizando la técnica de la lexicometría aplicada a diferentes temáticas, ver, por ejemplo, Bautista et. al, 2006, o Boltansky y Chiapello, 2007; para el uso del análisis factorial de correspondencias, ver Romeu, 1991).

Partiendo de una hipótesis previa sólida y una ejecución metodológica adecuada, la filosofía exploratoria de la lexicometría y del análisis de correspondencias "no debe entenderse sólo como mera descripción, ya que estas técnicas posibilitan el análisis, la comprobación y verificación de hipótesis previas" (Baptista y Sureda, 1987, p.173); es decir, estas técnicas permiten hacer ciertas generalizaciones en base a hipótesis previas fundamentadas -es por ello que en este trabajo no operamos de forma inductiva, como suele realizarse en otros trabajos que aplican este tipo de metodología, sino de forma deductiva, definiendo previamente las categorías que son importantes para defender las hipótesis planteadas, aspecto que ha de tenerse en cuenta al compararse con otros estudios que utilicen estos mismos métodos. Sin embargo, la fuerza del análisis lexicométrico reside tanto en la calidad de la justificación teórica y en la plausibilidad de las hipótesis previas al análisis, como en la correcta selección de textos representativos que definan bien cada uno de los grupos a comparar -lo cual garantiza, hasta cierto punto, la generalización-, así como en la correcta confección de los diccionarios y la rigurosa depuración de los datos. Además, es necesario complementar este análisis con otros, como el análisis de correspondencias, el análisis de textos y el análisis contextual de términos, con el fin de entrar en profundidad 
en el corpus de datos y eliminar así sus inevitables ambigüedades y el ruido derivado del análisis terminológico.

\section{Método}

\section{Participantes}

La muestra se compone de un total de seis libros de AA, cuatro libros y dos artículos científicos de PP, y cuatro manuales de PCC. Para la selección de los textos se controlaron un serie de variables con el fin de asegurar la representatividad de los mismos, tales como su nivel de impacto, su índice de popularidad, su volumen de ventas, su uso como manual de referencia, el número de citas/referencias o la cantidad de tiempo que han permanecido en el mundo editorial (años transcurridos desde su publicación, número de ediciones, etc.). También se tuvo en consideración que no se repitieran los autores, así como que hubiera representantes españoles e hispanohablantes en cada una de las categorías.

Para llevar a cabo el ANOVA y con el fin de igualar cada uno de los libros en unidades comparables, cada uno de ellos fue dividido en subconjuntos aleatorios de veinte mil palabras. Según el número total de palabras de cada libro, se pudieron realizar entre dos y seis divisiones. En la Tabla 1 se presentan los libros y artículos utilizados por cada uno de los tipos de literatura, así como el número de divisiones que aceptó cada uno de ellos para el ANOVA -nótese que el $N=51$.

Tabla 1. Libros (L) y artículos (A) utilizados, así como el número de divisiones que permitieron (entre paréntesis). Ordenados por grupos y por orden alfabético según autores.

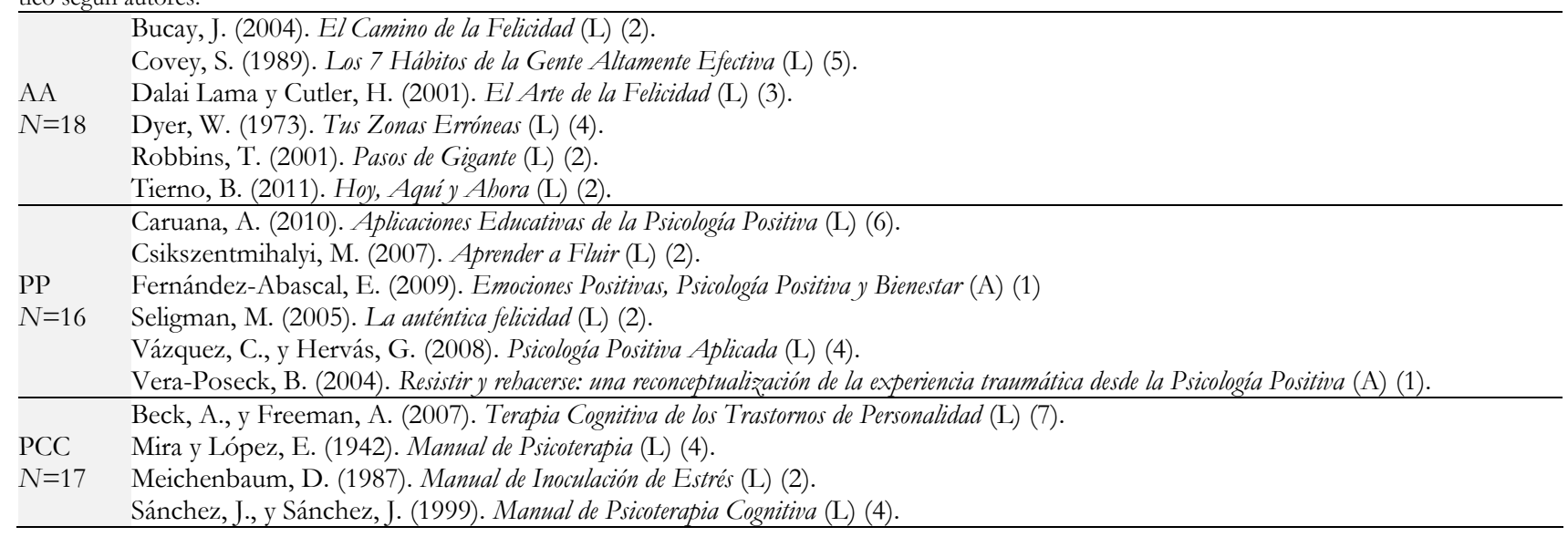

\section{Variables}

Las categorías de autoconocimiento, autocontrol, autocultivo y autodeterminación están representadas a través de un diccionario de términos previamente confeccionado siguiendo un criterio teórico y semántico. Cumpliendo con los requisitos metodológicos para el análisis lexicométrico y de correspondencias (Greenacre, 2008), fueron elaboradas categorías excluyentes y los más exhaustivas posible. Con el fin de no sobreestimar las frecuencias de algunas de estas categorías, no se contemplan en el diccionario aquellos términos que aún definiendo bien alguna de ellas se comprobó que resultaban demasiado problemáticas debido a su particular polisemia - por ejemplo, aunque para la categoría de "autodeterminación" son importantes sinónimos de "meta", fue descartada la palabra "objetivo" por referirse tanto a metas y fines como a una idea de verdad determinada; en cuanto a la palabra "fin", mediante un diccionario de exclusión se descartaron aquellas expresiones que no indicaran metas, tales como "a fin" o "con el fin". Para determinar la ambigüedad de los términos se recuperó el contexto discursivo de cada uno de los términos utilizados mediante la técnica KWIC que ofrece el programa WordStat 6.1.
El resto de nombres y adjetivos que no eran inicialmente relevantes para el análisis fueron analizados con el índice "TFxIDF" que proporciona WordStat 6.1. el cual indica cuán discriminativo resulta un término para un determinado grupo dado el conjunto de textos introducido. Así, cuando el término no era relevante para el análisis y presentaba un índice TFxIDF no significativo, era introducido en una categoría de "irrelevantes". Esta categoría mostró no ser discriminativa para ningún tipo de literatura -en el análisis de correspondencias, por ejemplo, se situaba en el punto $0.0 \mathrm{del}$ eje de clasificación-. En cambio, cuando algún término sí era significativo, y por tanto discriminativo, se introducía a posteriori dentro de una categoría temática coherente y se contemplaba dentro del análisis. Así, por ejemplo, las categorías de vocabulario de tipo "metodológico" o de tipo "médico" fueron construidos a posteriori, primero siguiendo el criterio de este índice y, posteriormente, un criterio semántico -se añadieron todos aquellos términos que denotaran categorías o jerga médica.

Para incrementar la validez interna de las categorías del diccionario y permitir una mayor sensibilidad tanto en el análisis como en las conclusiones, todas las categorías principales -autoconocimiento, autodeterminación, autocultivo y 
autocontrol- fueron divididas en subcategorías, permitiendo con ello concretar y definir en mayor profundidad el contenido psicológico de las categorías principales. Además, se añadieron al estudio otras categorías relevantes, con dos propósitos principales: primero, aportar mayor exhaustividad y riqueza al análisis de los libros, permitiendo abarcar una mayor cantidad de términos con posible contenido pertinente para realizar comparaciones entre los distintos tipos de literatura. En la Tabla 2 se presentan y ejemplifican las categorías y subcategorías analizadas.

Tabla 2. Categorías y subcategorías pertenecientes al individualismo "positivo", al vocabulario psicológico típico empleado y al tipo de retórica ejercida. Presentamos también ejemplos de los términos que componen cada una de ellas. En el análisis se utilizaron tanto estos términos como derivados y sinónimos suyos.

\begin{tabular}{lll}
\hline Categorías & Subcategorías & Ejemplos de términos \\
\hline Autoconocimiento & $\begin{array}{l}\text { Espacio psíquico } \\
\text { Actitudes positivas }\end{array}$ & $\begin{array}{l}\text { subjetividad, interior, propio, uno mismo, mente } \\
\text { optimismo, pesimismo, persistencia, confianza, negativo }\end{array}$ \\
\hline Autocontrol & $\begin{array}{l}\text { Locus de control interno } \\
\text { (Auto)motivación }\end{array}$ & $\begin{array}{l}\text { autocontrol, responsabilidad, poder, autonomía, elección } \\
\text { meta, motivación, fin, intención, interés }\end{array}$ \\
\hline Autocultivo & Cambio personal & cambiar, adaptar, transformar, moldear, aprender \\
\hline Autodeterminación & Mejora y crecimiento personales & desarrollar, crecer, mejorar, perfeccionar, potenciar \\
\hline \multirow{2}{*}{ Voc. psicológico "Básico" } & Bienestar y salud propios & bienestar, satisfacción, felicidad, salud, placer \\
& Logro y éxito propios & éxito, logro, ganar, conseguir, alcanzar \\
\hline Voc. Clínico & Pensamientos y cognición & pensamiento, idea, creencia, atribución, concepción \\
Voc. Médico & Emociones y estados de ánimónónimo, humor, alegría, risa \\
Voc. Metodóógico & Conductas y comportamientos & conducta, comportamiento, pauta, hábito, habilidad \\
\hline \multirow{2}{*}{ Retóricas de la verdad } & Clínico & trastorno, ansiedad, depresión, diagnóstico, paciente \\
& Médico & amígdala, cerebral, fármaco, médico, sustancias \\
\hline
\end{tabular}

\section{Procedimiento}

Para la confección del diccionario léxico se contó con tres psicólogos doctores y expertos conocedores de ambos tipos de literatura. Tras una primera fase de deliberación y de discusión sobre los términos que compondrían cada una de las categorías, se llevó a cabo una segunda fase de eliminación de aquellos términos que eran semánticamente ambiguos, como ha sido señalado.

Para el análisis lexicométrico se contabilizaron las frecuencias relativas para cada texto y tipo de literatura. Con el fin de que este porcentaje relativo se realizara sobre el total de los términos con contenido semántico -nombres comunes, adjetivos, nombres adjetivados y verbos-, todos los determinantes, conjunciones, artículos, pronombres y preposiciones fueron desechados del cómputo global. Para eliminar tales términos se utilizó el probado diccionario de exclusión desarrollado por Landauer y Dumais (1997). Además, se retiraron del análisis los apartados de referencias, los prólogos de otros autores y las notas a pie de página de todos los textos. Todo este procedimiento dejó un total de 198234 nombres, adjetivos, nombres adjetivados y verbos analizables en el caso de la AA, un total de 192183 en el caso de la PP, y un total de 201533 en el caso de la PCC.

Para comprobar si las diferencias entre los distintos tipos de literatura en cada una de las subcategorías de estudio eran significativas, se ejecutó la técnica ANOVA. Primero se comprobó la hipótesis de homocedasticidad con el estadístico de Levene. En todas las categorías, excepto en la de "cambio personal", dicho estadístico resultó ser significativo, por lo que se procedió a comprobar la diferencia de medias con el estadístico Games-Howell -en "cambio personal", sin embargo, se comprobó con el estadístico $F$.

Por último, con el fin de examinar cómo las distintas subcategorías de análisis se distribuyen en función de los tipos de literatura, se llevó a cabo un análisis de correspondencias, lo cual permite la clasificación factorial de todo el corpus de datos introducido en función de los tipos de literatura y de las subcategorías introducidas.

\section{Resultados}

\section{Descripción general de Tablas y Figuras}

La Tabla 3 ofrece dos tipos de información: el primer bloque de columnas (1) indica el porcentaje total relativo de términos de cada una de las categorías analizadas por tipo de literatura; el segundo bloque de columnas (2) ofrece el nivel de significación resultado del contraste de medias realizado en cada una de las subcategorías analizadas por tipo de literatura.

Como puede apreciarse, ninguna de las categorías y subcategorías del individualismo "positivo" - excepto una de estas últimas - arroja diferencias significativas entre las AA y la PP, mientras que todas ellas -excepto esta mismas- se muestran significativamente diferentes entre estos dos tipos de literatura y la PCC. También puede evidenciarse cómo tampoco existen diferencias significativas entre la PP y la AA en cuanto al tipo de vocabulario psicológico "básico", "clínico" o "médico" se refiere, siendo la única diferencia entre 
ambos la mayor cantidad de vocabulario "metodológico" por parte de la PP. Respecto a los cuatro tipos de vocabulario, tanto la PP como la AA guardan diferencias significativas con la PCC excepto en dos comparaciones particulares: PP y PCC en vocabulario metodológico, y AA y PCC en el vocabulario referente a "emociones y estados de ánimo". Respecto al tipo de retórica utilizada, vemos que cada tipo de literatura se caracteriza por utilizar en mayor medida una distinta a los demás: la AA utiliza un mayor tipo de retórica universalista o absolutista -sus guías de comportamiento se dicen basar en verdades en sí-, la PP utiliza un mayor tipo de retórica científica -sus técnicas se dicen basar en la evidencia empírica- y la PCC un mayor tipo de retórica tanto científica como de la practicidad -se defiende que sus técnicas están probadas y que son eficientes y útiles. En total, vemos como estas 9 categorías permiten cubrir entre el 18\% y el $23 \%$ del total de términos presentes en cada tipo de literatura.

Tabla 3. 1) Porcentaje relativo de términos por cada uno de los tipos de literatura en cada categoría y subcategoría. 2) Nivel de significación de la diferencia de medias entre los tipos de literatura en cada una de las categorías obtenido mediante el estadístico de contraste Games-Howell. Las comparaciones significativas $(p<.05)$ están en cursiva.

\begin{tabular}{|c|c|c|c|c|c|c|c|}
\hline & & \multicolumn{3}{|c|}{1.} & \multicolumn{3}{|c|}{2.} \\
\hline & & $\mathrm{AA}$ & $\mathrm{PP}$ & PCC & AA-PP & AA-PCC & PP-PCC \\
\hline \multirow[t]{3}{*}{ Autoconocimiento } & Espacio psíquico & 1.7 & 1.5 & 1.1 & .97 & .00 & .00 \\
\hline & Actitudes positivas & 1.1 & 1.6 & 0.5 & .12 & .00 & .00 \\
\hline & $\Sigma$ & 2.8 & 3.1 & 1.6 & & & \\
\hline \multirow[t]{3}{*}{ Autocontrol } & Locus de control interno & 1.9 & 2.3 & 1.3 & .09 & .01 & .00 \\
\hline & (Auto)motivación & 1.4 & 1.4 & 1 & .96 & .00 & .04 \\
\hline & $\sum$ & 3.3 & 3.7 & 1.4 & & & \\
\hline \multirow[t]{3}{*}{ Autocultivo } & Cambio personal & 0.9 & 0.9 & 0.8 & .99 & .46 & .4 \\
\hline & Mejora y crecimiento personales & 1.5 & 2 & 0.8 & .00 & .00 & .00 \\
\hline & $\Sigma$ & 2.4 & 2.9 & 1.6 & & & \\
\hline \multirow[t]{3}{*}{ Autodeterminación } & Bienestar y salud propios & 1.2 & 1.7 & 0.4 & .44 & .00 & .00 \\
\hline & Logro y éxito propios & 1 & 0.7 & 0.4 & .28 & .04 & .02 \\
\hline & $\Sigma$ & 2.2 & 2.4 & 0.8 & & & \\
\hline \multirow[t]{3}{*}{ Vocabulario Psicológico "Básico" } & Pensamientos y cognición & 1.2 & 1.3 & 2.5 & .97 & .00 & .00 \\
\hline & Emociones y estados de ánimo & 1.6 & 2.1 & 1.4 & .40 & .39 & .09 \\
\hline & Conductas y comportamientos & 0.6 & 0.4 & 1.1 & .43 & .03 & .00 \\
\hline Vocabulario de tipo Clínico & Clínico & 0.3 & 1.1 & 6 & .12 & .00 & .00 \\
\hline Vocabulario de tipo Médico & Médico & 0.2 & 0.4 & 0.7 & .30 & .00 & .06 \\
\hline $\begin{array}{l}\text { Vocabulario de tipo } \\
\text { Metodológico }\end{array}$ & Metodológico & 0.9 & 1.8 & 2 & .00 & .00 & .82 \\
\hline \multirow[t]{3}{*}{ Retóricas de la verdad } & Científica & 0.8 & 1.6 & 1.1 & .00 & .04 & .01 \\
\hline & Universalista & 0.9 & 0.3 & 0.3 & .00 & .00 & .98 \\
\hline & Practicidad & 1 & 1.1 & 1.6 & .97 & .05 & .04 \\
\hline$\sum$ & & 18 & 22 & 23 & & & \\
\hline
\end{tabular}

Utilizando la técnica KWIC, las Tablas 4, 5, 6 y 7 recogen fragmentos de cada una de las cuatro categorías del individualismo "positivo" -autocontrol, autoconocimiento, autocultivo y autodeterminación, respectivamente- por cada tipo de literatura. Utilizando el mismo término clave, cada una de las tablas recupera, a modo de ejemplo, el contexto semántico de los términos, permitiendo con ello desambiguar su uso y comparar el significado más preciso del mismo entre los distintos tipos de literatura. Para un mejor seguimiento del análisis, cada una de estas tablas se presenta insertada en cada uno de sus respectivos apartados, presentados a continuación.

La Figura 1 muestra el resultado del análisis de correspondencias llevado a cabo. Como vemos, tanto la AA como la PP se sitúan muy próximos entre sí y en torno a las subcategorías psicológicas del individualismo "positivo" -todas ellas dentro del óvalo azul. También podemos observar que dentro de este modelo, ciertas subcategorías tienden a caracterizar más un tipo de literatura que otra. Por su parte, la PCC se sitúa en la zona opuesta de la distribución, alejada tanto de la AA como de la PP, estando poco caracterizada por las categorías del individualismo "positivo" y mucho por un tipo de vocabulario de tipo clínico, médico, cognitivo, conductual y, en menor medida, metodológico. También observamos cómo cada uno de los tipos de retórica es más característica de un tipo de literatura. En cuanto a la categoría de términos "irrelevantes", ésta tiende a quedar situada cerca del punto 0.0 del eje de coordenadas, indicando que no sirve para discriminar entre los diferentes tipos de literatura -el porcentaje relativo de esta categoría, que incluye nombres propios y comunes, adjetivos y verbos no discriminativos según el índice TFxIDF, es de alrededor de un 45\% para cada uno de los tipos de literatura. 


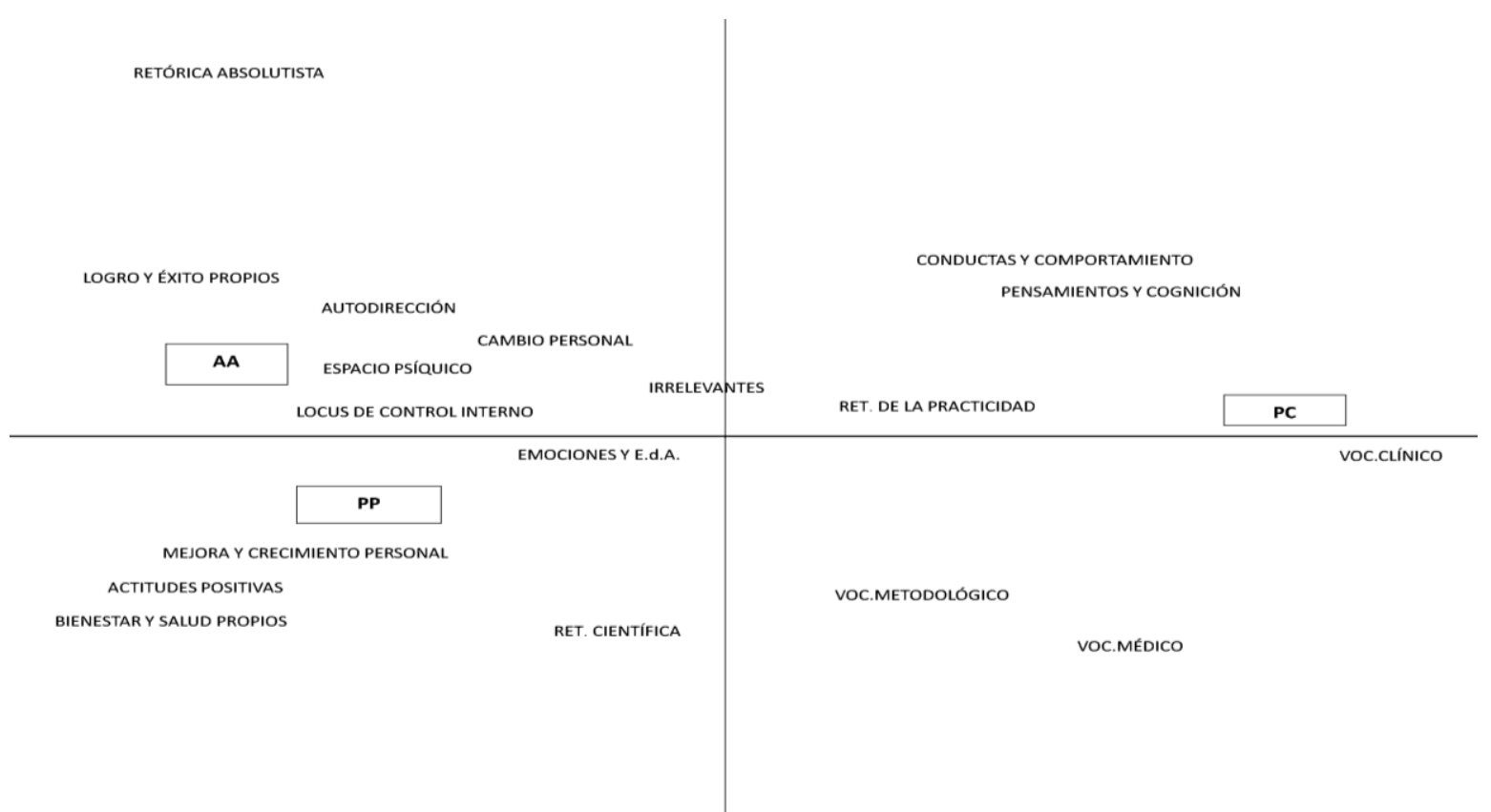

Figura 1. Análisis de Correspondencias con todas las categorías de análisis -dentro del área azul, las categorías psicológicas características del individualismo "positivo".

\section{Descripción y análisis por categorías}

\section{Autocontrol}

Como puede apreciarse en los resultados, una de las categorías psicológicas más importantes tanto para la AA como para la PP es la de "autocontrol", algo que es enormemente congruente con la literatura escrita al respecto véanse, por ejemplo, Cabanas, 2011a, 2011b; Cabanas y Sánchez, 2012; Ehrenreich, 2009; Illouz, 2010; Rimke, 1997; 2000. En torno al individualismo "positivo" gravita la idea de que la felicidad depende enteramente de uno mismo, esto es, de una voluntad correctamente dirigida hacia el autocontrol y hacia la autorregulación de nuestras actitudes, emociones y pensamientos, así como del manejo inteligente de nuestras atribuciones cognitivas y de nuestras relaciones sociales. Para el individualismo "positivo" uno es feliz siempre de "dentro afuera": la felicidad del hombre está en sí mismo, insistiendo en la completa responsabilidad del individuo sobre su felicidad y su sufrimiento, su salud y su enfermedad, sus éxitos y sus fracasos. Si la responsabilidad es enteramente del individuo, entonces las circunstancias de cada cual apenas tienen importancia, y cuando la tienen, en el grado que sea según diversos autores -aunque siempre jugando un papel muy secundario-, son siempre entendidas de "dentro afuera", como decimos, esto es, de forma relativa a la interpretación que de las mismas hace el propio sujeto.

Un ejemplo de ello es la denominada "fórmula de la felicidad" - $\mathrm{F}($ felicidad $)=\mathrm{H}($ herencia genética $)+\mathrm{V}($ voluntad $)+\mathrm{C}$ (circunstancias)"-, propuesta por Martin Seligman y defendida por la gran mayoría de los psicólogos positivos y de los coachers, la cual establece que los factores genéticos expli- can un $50 \%$ de la felicidad de los individuos, y que otros factores individuales como la voluntad propia, el control de los pensamientos y las emociones, y una actitud positiva, explican el 40\% (Lyubomirsky, Sheldon y Schkade, 2005, Seligman, 2005). En cambio, "factores circunstanciales" como pudieran serlo la situación de desempleo, una ruptura de pareja, una enfermedad crónica o que te toque la lotería, por ejemplo, únicamente explican un 10\% del total según esta propuesta. Dentro del mundo de la AA encontramos múltiples ejemplos similares a la propuesta de Seligman. Por ejemplo, en el libro "El Camino a la Felicidad" publicado por Jorge Bucay (2000. p.5) se defiende lo siguiente:

Intentaré mostrar que cada uno es portador del principal - aunque no único- determinante de su nivel de felicidad. Un factor variable de individuo en individuo, y cambiante en diferentes etapas de una misma persona, al que voy a llamar, caprichosamente, "factor F". Aun a riesgo de simplificarlo demasiado, lo defino básicamente como la suma de tres elementos principales: a) Cierto grado de control y conciencia del intercambio entre nosotros y el entorno; b) el desarrollo de una actitud mental que nos permita evitar el desaliento; c) el trabajo para alcanzar sabiduría.

Como se aprecia en los resultados respecto a la subcategoría de "locus de control interno" -Tabla 3-, el lenguaje del autocontrol, la autorregulación, la responsabilidad, la voluntad y la autonomía es muy relevante tanto para la AA como para la PP $-2.3 \%$ y $1.9 \%$, respectivamente. Esto es indicador de qué tipo de caracterización psicológica es definitorio del modelo de sujeto que ambos tipos de literatura defienden, para los cuales el individuo es el completo responsable y 
agente de lo que le acontece, de lo que piensa y de cómo se siente: es el propio sujeto quien gestiona sus propios problemas y quien busca las posibles soluciones. No obstante, aunque la diferencia entre ambos tipos de literatura no es significativa -.09-, conviene señalar que la AA tiende a hacer un mayor uso de esta terminología que la PP. Esto es debido, principalmente, a que en la AA predomina la continua presentación de recetarios comportamentales del tipo "cómo hacer -"how tos"- sobre la teorización de los mismos, siendo esto último más característico de la PP.

En cuanto a la PCC, los términos relativos al locus de control interno se utilizan de forma significativamente menor que en el caso de la AA y la PP. Destacamos dos razones principales. Primero, porque la PCC otorga mucho más peso explicativo a elementos estructurales -factores ambientales y circunstanciales- e interpersonales a la hora de explicar las causas del comportamiento de los individuos, así como a la hora de atribuir responsabilidad al individuo por sus problemas, pensamientos, afectos, etc. Segundo, porque las técnicas psicológicas que provee la PCC no son de tipo autoaplicado, esto es, no están dirigidas al propio individuo o usuario final, sino a la formación de expertos psicoterapeutas responsables de adecuar la aplicación de las técnicas a los problemas concretos de estos usuarios/pacientes/clientes finales -ver Tabla 4.

Una explicación análoga a esta sirve para entender los datos relativos a la subcategoría de "automotivación". Términos como "metas", "objetivos", "intenciones", "intereses", "motivación", etc., son también utilizados de forma significativamente mayor tanto por la AA como por la PP $1.4 \%$ y $1.4 \%$, respectivamente, frente al $1 \%$ de la PCC. Ambas ponen especial énfasis en que es el propio individuo el encargado de planificar la mejor forma de guiarse hacia la realización de sus propios fines e intereses, de priorizar su persecución y de perseverar en el esfuerzo de alcanzarlos, algo esencial en las ideas de la autorrealización y el florecimiento personal (Seligman, 2011) que son comunes para PP y AA.

En general, la categoría de "autocontrol" es más definitoria de la PP y de la AA, que de la PCC. Esto se aprecia también en el análisis de correspondencias -AC- de la Figura 1. el cual indica, además, que si bien tanto la AA como la PP insisten en el locus de control interno y en la necesidad de la automotivación, ambas son algo más características de la AA. En el caso de la PCC, la categoría de "autocontrol" se utiliza para denotar aspectos diferentes a la PP y la AA, como dijimos, algo que también podemos comprobar recuperando el uso de los términos en su contexto -ver Tabla 4Para la PCC, el "autocontrol" no denota tanto la asunción psicológica de que es el individuo el centro sobre el cual giran los acontecimientos que le rodean, el origen causal de todo lo que afecta y le define, algo principal para la PP y la AA. En el caso de la PCC, esta categoría se utiliza para denotar la necesidad del individuo de adoptar un papel de implicación activa en el proceso terapéutico, gracias al cual se facilita la consecución de los objetivos de la terapia, los cua- les están orientados hacia la integración contextual y ambiental -amigos, familia, trabajo, etc. - del individuo, y hacia la restitución de su adecuado funcionamiento cognitivo y emocional; no la utiliza, al contrario que la PP y la AA, como un aspecto vertebral para definir conceptual y moralmente el funcionamiento natural del individuo.

Tabla 4. Ejemplos de "autocontrol" obtenidos recuperando el contexto de los términos clave. Cursivas nuestras.

Autoayuda:

Somos responsables de nuestras propias vidas; nuestra conducta es una función de nuestras decisiones, no de nuestras condiciones (Covey, 1997, p.42).

El secreto para desatar tus fuerzas es establecer objetivos interesantes que despierten tu creatividad y enciendan tu pasión. Elige ahora mismo tus objetivos. Analiza a fondo todo lo que vale la pena para ti. Y elige el objetivo que más te inspire, el que te hará levantar te pronto por la mañana y acostarte tarde por la noche (Robbins, 2001).

Psicología Positiva:

Los trabajadores intentar conseguir sus objetivos por medio de un sentido de agencia o control personal que les provee de la fuerza de voluntad necesaria para conseguir sus objetivos. Los trabajadores con altas dosis de esperanza están también motivados para conseguir objetivos a través del desarrollo de planes para conseguir lo que quieren. Además, si alguno de estos planes falla, tienen la capacidad de desarrollar planes alternativos" (Vázquez y Hervás, 2009).

La buena noticia sobre las circunstancias es que algunas generan mayor felicidad. La mala es que cambiar tales circunstancias suele ser poco práctico y costoso (Seligman, 2005).

Psicoterapia Cognitivo-Conductual

No hay ningún recurso mágico para controlar y cuestionar pensamientos, aprender a relajarse, etcétera, y los clientes suelen ser mucho más aquiescentes si tienen un papel activo en la adaptación de las técnicas estándar a sus propias necesidades y preferencias (Beck y Freeman, 2007).

La categoría psicológica de autocontrol, tal y como está conceptualizada por el individualismo "positivo", juega un papel crucial en la idea de felicidad. Lo verdaderamente importante para la felicidad, dicen tanto psicólogos positivos como escritores de autoayuda, no es cambiar las condiciones, sino cambiar el modo en cómo cada cual interpreta las mismas: la felicidad es cuestión del cristal con el que miramos el mundo, es decir, de cómo el sujeto interpreta la realidad social y sus condiciones vitales en función de sus actitudes personales. Esta defensa de una felicidad de "dentro afuera", fruto del control de los propios estados emocionales y de la interpretación que los individuos hagan del mundo, y de exclusiva responsabilidad personal, no sólo vertebra en buena medida el discurso de la literatura sobre la felicidad y el bienestar -tanto académica como popular- sino también el discurso cotidiano que los individuos emplean para explicar tanto sus comportamientos y sentimientos como los de los demás. 


\section{Autoconocimiento y tipos de vocabulario}

Otra de las importantes semejanzas esperables entre la PP y la AA estribaría en el tipo de vocabulario que ambas proveen para tematizar el interior del sujeto con el fin de que éste pueda relacionarse consigo mismo identificando qué es aquello que debe conocer y someter a control, cambio y mejora. A este respecto, una de las peculiaridades del individualismo "positivo" frente a otros tipos de discursos psicológicos sobre el individuo es la tendencia a usar un lenguaje genérico, poco específico y coloquial sobre el interior. Para analizar esto fueron creadas dos subcategorías que recogen terminología de este tipo: la de "espacio psíquico", representado por términos como "mente", "conciencia", "subjetivo", "propio", "interior", "uno mismo", etc.; y la de "actitudes positivas": "optimismo", "pesimismo", "persistencia”, "confianza", "negativo", etc. Como contraposición al contenido genérico de esta categoría se analizó un tipo de vocabulario que denota más especificidad psicológica a la hora de definir el interior, como es el caso de las subcategorías "pensamientos y cognición", "emociones y estados de ánimo", "conductas y comportamientos", "vocabulario clínico" y "vocabulario médico", las cuales nos permiten profundizar en el análisis de las diferencias y semejanzas entre PP, AA y PCC.

En cuanto a la terminología genérica del interior, en la Tabla 3 se puede observar el elevado uso que tanto en el discurso de la PP como en el de la AA hacen de términos relacionados con la subcategoría "espacio psíquico"-1.5\% y $1.7 \%$, respectivamente-, y la subcategoría "actitudes positivas" $-1.6 \%$ y $1.1 \%$ respectivamente. En ambas categorías no existen diferencias significativas entre ambos tipos de literatura. Por el contrario, la PCC presenta significativamente menores frecuencias en ambas subcategorías $-1.1 \%$ y $0.50 \%$, respectivamente- que la AA y la PP.

Tanto la PP como la AA hacen suyo el vocabulario de las "actitudes positivas" con el fin de acotar su objeto psicológico de estudio, así como para suplir la menor presencia de una terminología más técnica y específica sobre el interior. Este mayor uso de la terminología de las "actitudes positivas" por parte de la PP y la AA es todavía más relevante para la PP que para la AA, como indica el AC de la Figura 1. Esto es debido a que mientras que la PP explicita marcos teóricos para explicar la relevancia que las "actitudes positivas" tendrían sobre el comportamiento de los individuos, la segunda carece de esta pretensión teórica.

En cuanto al uso de un tipo de vocabulario psicológico más específico, mientras que el lenguaje de las emociones es utilizado por todos los tipos de literatura de forma similar aunque no existen diferencias significativas es un tanto más abundante en el caso de la PP-, la PCC suele optar por utilizar el lenguaje algo más técnico de las pautas de comportamiento $-1.1 \%$ - y de los procesos cognitivos asociados a las mismas $-2.5 \%-$ de forma significativamente mayor que la PP y la AA. Además, la PCC posee una jerga técnica propia, en este caso de tipo clínico $-6 \%$-, de la que carecen las otras dos, y que es enormemente definitorio del objeto de estudio de la PCC. Nótese también que la PP utiliza un 1.1\% de terminología de tipo clínico. Sin embargo, no la utiliza tanto para definir su objeto de estudio como para reivindicar que su campo de interés supone una alternativa a este tipo de psicología clínica, de la cual predican que tiende a pasar por alto el estudio de las personas sanas y su necesidad de mejorar y aumentar su felicidad (Seligman y Csikszentmihalyi, 2000). En cuanto al uso de un vocabulario de tipo médico y biológico, tanto PP como PCC hacen cierto uso del mismo $-0.4 \%$ y $0.7 \%$, respectivamente-, el cual ambos utilizan de forma esporádica para justificar la base biológi$\mathrm{ca} /$ neurológica de sus respectivas posturas psicológicas.

Los datos obtenidos para la categoría de "autoconocimiento" son congruentes con aquellos presentados por la literatura existente al respecto. Según la misma, sin una terminología psicológica técnica sobre el interior que establezca una fuerte diferencia entre la PP y la AA, esta conceptualización genérica del interior confiere al discurso sobre el sujeto una mayor adaptabilidad e inclusividad para un amplio conjunto de personas, problemas y situaciones. Como dice Eva Illouz (2010), cuanto más genérico es el discurso psicológico, más móvil y flexible es, pudiendo ser adaptado a una variedad de situaciones tal que es capaz de explicar la particularidad individual a la vez que permite compartirla con otros mediante este lenguaje genérico. Esta falta de especificidad, además, es de especial utilidad cuando es el individuo mismo y no otro - una autoridad exterior, por ejemplo, como es el caso del psicoterapeuta en la psicoterapia- quien tiene que indagar, vigilar y censurar constantemente su propio "interior”. Es el propio individuo la persona más indicada para determinar cuáles son la verdaderas causas de sus pensamientos y comportamientos, así como cuáles son las prácticas más idóneas para corregirlos y/o dirigirlos hacia la consecución del bienestar; y para ello no requiere un vocabulario ni variado ni específico sobre su interior. Digamos que es el individuo el propio terapeuta de sí mismo, algo que queda patente tanto en las técnicas que ofrecen la PP y la AA como en las que ofrece el coaching (véanse, por ejemplo, Seligman, 2002; Vázquez y Hervás, 2008, 2009; Linley y Joseph, 2004; Biswas-Diener y Dean, 2007; Lyubomirsky, 2007).

$\mathrm{Al}$ margen de esta mayor o menor generalidad, el discurso del individualismo "positivo" enfatiza que las causas de la felicidad son internas y que, por tanto, si su secreto reside en el interior, es necesario que el individuo se conozca bien para encontrar las claves de la felicidad y del desarrollo personal -ver Tabla 5. Esta hipertrofia del espacio interior y el constante uso de la terminología de las actitudes positivas enfatizan aún más tanto la idea de responsabilidad propia del individuo sobre su propia felicidad, como la reducción de ésta a factores emocionales y cognitivos: para el individualismo "positivo" la felicidad es, como decíamos, una cuestión de interpretación del mundo y de las circunstancias que nos rodean siempre de "dentro afuera", desde el individuo hacia el exterior, y no tanto al contrario. Conocer qué esperamos del mundo -optimismo, esperanza- y de nosotros mismos 
-autoconfianza-, así como descubrir cuáles son nuestras virtudes y nuestras fortalezas personales para poder desarrollarlas y ponerlas en práctica son aspectos esenciales tanto para la PP (Peterson y Seligman, 2004), como para la AA y el coaching (Biswas-Diener y Dean, 2007).

Tabla 5. Ejemplos de "autoconocimiento" obtenidos recuperando el contexto de los términos clave. Cursivas nuestras.

Autoayuda:

Debemos ser conscientes de los efectos beneficiosos de las emociones y comportamientos positivos; ello nos llevará a cultivar, desarrollar y aumentar esas emociones, por difícil que sea. Tenemos una fuerza interior espontánea. A través de este proceso de aprendizaje, del análisis de pensamientos y emociones, desarrollamos gradualmente la firme determinación de cambiar, con la certidumbre de que tenemos en nuestras manos el secreto de nuestra felicidad, de nuestro futuro, y de que no debemos desperdiciarlo (Dalai-Lama y Cutler, 2001).

Escribe un diario donde vayas anotando tus comportamientos autodestructivos, y apunta no sólo tus actos sino también lo que sentías cuando te comportabas de esa manera. Durante una semana apunta en una libreta la hora exacta, la fecha y la ocasión en que usas cualquiera de los "Yo soy" autodestructivos, y esfuérzate por disminuir el número de apuntes (Dyer, 1996)

Psicología Positiva:

Yo hago lo siguiente: (...) en una escala del 1 al 10 -de pésimo a perfecto- valoro mi satisfacción con la vida en cada uno de los ámbitos que evalúo, y escribo un par de frases que los resuman. Estos ámbitos, que pueden ser distintos para cada persona, son los siguientes: Amor, Profesión, Finanzas, Juegos, Amigos, Salud, Creatividad, En conjunto. Utilizo otra categoría, Trayectoria, en la que analizo los cambios existentes de un año a otro y el comportamiento observado en éstos a lo largo de la década. Recomiendo este procedimiento a los lectores, pues sirve para concretar, deja poco margen al autoengaño e indica cuándo actuar. Parafraseando a Robertson Davies: "Valora tu vida una vez al año. Si descubres que no das el peso exacto, cambia de vida. Seguramente descubrirás que la solución está en tus manos" (Seligman, 2005).

\section{Autocultivo}

Para ser feliz, además de controlarse y de conocerse, el individuo ha de cuidar y de cultivarse a sí mismo con el fin de "florecer" (Seligman, 2011). Sin ello, la consecución de la felicidad y del éxito propio no está garantizada, pues la felicidad no es una meta que se alcance una sola vez y para siempre, sino que el individuo ha de estar continuamente buscando formas de cambio y de mejora personal para alcanzar e incrementar su felicidad.

Este tipo de autocultivo constante, pues, es otra de las categorías psicológicas que vertebran el individualismo "positivo": como se observa en la Tabla 3, AA y PP presentan un $2.4 \%$ y un $2.9 \%$ de términos dedicados a esta categoría, mientras que la PCC utiliza un 1.6\%. Sin embargo, cabría esperar, como de hecho ocurre, que dentro de esta categoría, la subcategoría de "cambio personal" fuera la que menos diferencia terminológica presente entre los tres tipos de literatura -no hay diferencias significativas entre ninguna de ellas, puesto que en todos ellos abunda terminología referida al cambio y la modificación debido a su carácter primordialmente práctico y de resolución de problemas personales. Por el contrario, es esperable que tanto en la PP como la AA, especialmente la primera, abunde el vocabulario relacionado con el "crecimiento y la mejora personales", aspecto que está íntimamente relacionado con la idea de felicidad que ambas comparten. Así, por un lado, no existen diferencias significativas entre los tres tipo de literatura respecto a la subcategoría de "cambio personal" $-\mathrm{AA}=0.9 \% ; \quad \mathrm{PP}=0.9 \%$; PCC $=0.8 \%$. Todo lo contario ocurre en el caso de "mejora y crecimiento personal" $-\mathrm{AA}=1.5 \% ; \mathrm{PP}=2 \% ; \mathrm{PP}=0.8 \%$, donde la AA y la PP utilizan entre dos y tres veces más este tipo de terminología que la PCC. Respecto al AC -Figura 1-, ambas subcategorías aparecen como más definitorias de la AA y de la PP que de la PCC, siendo más relevante la de "cambio personal" para la AA y la de "mejora y crecimiento personales" para la PP -esto último también se refleja en la significación de la diferencia de medias entre ambas.

Sin embargo, si analizamos el significado concreto de cada una de las subcategorías por separado, así como la relación teórica entre ellas, observamos que PP y AA difieren de la PCC en el modo de entender las mismas. Así, mientras que el discurso de la PP y de la AA tiende a relacionar el cambio con el crecimiento personal y con la búsqueda de la felicidad, la PCC lo relaciona con metas completamente distintas - ver Tabla 6. Así, mientras que los tres tipos de literatura entienden que para superar determinados obstáculos el individuo ha de esforzarse por cambiar -implicándose activamente en el tipo de propuestas que se derivan de cada tipo de literatura-, para la PP y la AA el cambio es insuficiente si no va orientado hacia el crecimiento y el florecimiento del "uno mismo". Así como para la PCC lo saludable es sinónimo de adaptación y de superación de aquello que hacía sufrir al individuo, para el individualismo "positivo" lo saludable es sinónimo de potenciación de la felicidad y de florecimiento individual: tanto para la AA como para la PP, cambio implica siempre mejora, necesariamente, algo que reconocen tanto escritores de autoayuda como psicólogos positivos y que se defiende como un rasgo diferencial frente a la psicoterapia "tradicional" (Seligman y Csikszentmihalyi, 2000). De hecho, desde su fundación la PP ha ido cada vez haciendo más énfasis en la importancia del crecimiento personal para la felicidad: como el mismo Seligman indica, "ahora considero que el núcleo de la psicología positiva es el bienestar, que el patrón de oro para medir el bienestar es el crecimiento personal y que el objetivo de la psicología positiva es aumentar dicho crecimiento" (2011. p. 28). Además, tanto psicólogos positivos y escritores de autoayuda enfatizan que el trabajo sobre uno mismo no se acaba nunca: si las cosas te van bien tienes que seguir manteniendo la actitud positiva, y si ya has conseguido tus metas, no te has de conformar con ellas, señala Barbara Ehrenreich (2009) como característico de este discurso. 
Tabla 6. Ejemplos de "autocultivo" obtenidos recuperando el contexto de los términos clave. Cursivas nuestras.

\section{Autoayuda:}

Tu motivación puede provenir de un deseo de crecer y desarrollarte más que de un deseo de reparar tus deficiencias. Si llegas a reconocer que siempre podrás crecer, mejorar, desarrollarte, volverte cada vez más y más grande, ya es suficiente (...) La motivación del crecimiento y el desarrollo implica usar tu energía vital para alcanzar una mayor felicidad (Dyer, 1993).

\section{Psicología Positiva:}

No sentirse mal en la vida no debería ser suficiente. Debemos tener metas más ambiciosas... Es posible que nuestro paciente ya no tenga síntomas de depresión, de ansiedad o psicóticos. Pero, ¿está realmente bien?, ¿se siente en sintonía con la vida?, ¿puede desarrollar lo mejor de sí mismo...? (Vázquez y Hervás, 2009).

\section{Autodeterminación}

La categoría de autodeterminación que aquí defendemos como particular del individualismo "positivo" está principalmente ligada al ideal norteamericano del "self-made man", el cual enfatiza la idea de que es el individuo el dueño y el escritor de su propio destino, explicando que la motivación por el éxito y la salud, el esfuerzo individual y la iniciativa personal deben desarrollarse sin necesidad de apoyo o coerción externa. Esta categoría representa el horizonte moral al que constantemente apunta el sujeto del individualismo "positivo": bienestar, salud, éxito y logro son metas por las cuales el individuo se esfuerza y persevera en el autocontrol, por el cual atiende y escruta sus pensamientos y sus emociones, y por el cual invierte tiempo y dinero en sí mismo, aplicándose constantemente nuevas y mejoras de crecer y desarrollarse personalmente.

En la Tabla 3 apreciamos que esta categoría es especialmente relevante tanto en la AA y en la PP, entre las cuales, además, no se aprecian diferencias significativas en ninguna de las subcategorías. Por el contrario, las diferencias entre la PP y la AA respecto a la PCC sí lo son. Según los resultados obtenidos, AA y PP utilizan un $1.2 \%$ y un $1.7 \%$ del total de sus términos respectivamente para hablar de la consecución del "bienestar y la salud propios", y un total de $1 \%$ y $0.7 \%$ para hablar del "logro y el éxito personales". La PCC, sin embargo, muestra un total de un $0.4 \%$ de sus términos en ambas subcategorías.

Es importante destacar, no obstante, que los tres tipos de literatura enfatizan la consecución de metas y objetivos como parte importante de su vena interventiva, aplicada y práctica, pero la forma de tematizar estas metas es bien distinta-ver Tabla 7-, ya que mientras que la PCC destaca metas de tipo paliativo, adaptativo y de restauración del normal funcionamiento psicológico de los individuos, la PP destaca la consecución de metas internas como la felicidad y la salud -"gratificación", "bienestar", "satisfacción", "vitalidad", "plenitud"-, y la AA la idea del logro y el éxito propios "competición", "triunfo", "liderazgo", "reconocimiento", "éxito"-, algo que se aprecia en el AC de la Figura 1.
Tabla 7. Ejemplos de "autodeterminación” obtenidos recuperando el contexto de los términos clave. Cursivas nuestras.

Autoayuda:

La voluntad de vencer — de tener éxito, de dar forma a la propia vida, de tomar las riendas - sólo se puede dominar cuando sabes lo que quieres y crees firmemente que ningún problema, ninguna dificultad, ningún obstáculo te puede apartar de tu meta. Los obstáculos solo son incentivos para aumentar tu resolución de alcanzar tus objetivos (Robbins, 2001).

Psicología Positiva:

Otro aspecto de esta fortaleza [inteligencia personal] es encontrar los "espacios" adecuados para uno mismo, como por ejemplo situarse en entornos que maximicen las habilidades $e$ intereses personales. $¿ \mathrm{Ha}$ escogido un trabajo, sus relaciones íntimas y sus actividades de ocio de forma que le permitan utilizar sus mejores habilidades cada día, si es posible? ¿Le pagan por hacer lo que verdaderamente se le da mejor? La Organización Gallup descubrió que los trabajadores más satisfechos eran los que respondían afirmativamente a la pregunta: "¿Su trabajo le permite hacer lo que sabe hacer mejor todos los días?" Basta pensar en Michael Jordan, jugador de béisbol mediocre, que "se encontró a sí mismo" jugando al baloncesto (Seligman, 2005).

Psicoterapia Cognitivo-Conductual:

Los programas de control del estrés...deben fomentar una fórmula única o simple, o un enfoque a manera de recetario para afrontar el estrés. El individuo debe aprender a adaptar su estilo a las exigencias de la situación y a los contextos y objetivos cambiantes (Meichenbaum, 1987).

\section{Retóricas de la Verdad}

Una de las principales diferencias que cualquier lector vislumbra en la lectura de estos tres distintos tipos de literatura tiene que ver con la legitimidad y la autoridad que el individuo atribuye a los diferentes textos, así como con la veracidad que concede a los argumentos psicológicos que se despliegan en ellos. El tipo de retórica es un factor relevante que modula estos aspectos. Así, elementos como el lenguaje más o menos cercano con el que el autor escribe, la apelación a experiencias personales o a literatura especializada, o la cantidad de terminología técnica que se utiliza para desarrollar el argumento, influyen en la capacidad persuasiva y en el nivel de autoridad que el texto tiene sobre la credibilidad de los lectores. En la comparación de estos tres tipos de literatura hemos visto diferencias a este nivel.

Tanto la AA como la PP defienden la búsqueda de la felicidad como algo natural, inherente a la condición humana. Como intentamos defender, apelan a explicaciones sobre el comportamiento y a conceptualizaciones sobre la naturaleza psicológica del sujeto que son muy similares. La principal diferencia que surge de un análisis cuantitativo a nivel terminológico estriba en el uso que cada una de ellas hace de aquello que aquí hemos denominado como "retóricas de la verdad". De este modo, mientras que la literatura de autoayuda da por supuesta la veracidad de sus argumentos mediante una retórica apoyada en términos de verdades necesarias, es decir, de prejuicios sobre el funcionamiento humano que tanto autores como lectores dan por supuestos, la PP 
presenta sus argumentos mediante el abundante uso de terminología científica y metodológica.

Como puede apreciarse en la Tabla 3, la PP utiliza un $1.6 \%$ de terminología científica y un $1.8 \%$ de terminología metodológica, de forma significativamente mayor que la AA, que utiliza un $0.8 \%$ y un $0.9 \%$, respectivamente. PP y PCC comparten un uso similar de terminología metodológica, pero la PP hace un uso significativamente mayor de terminología científica que esta última $-1.6 \%$ y $1.1 \%$, respectivamente. Este resultado es compatible con la crítica de muchos autores hacia la PP cuando señalan que ésta se reviste de una profusa retórica científica -"demostración", "descubrimiento", "estudio", "evidencia", "datos", etc.- con el fin de demarcarse del marco cultural del que proviene (Christopher y Hickimbottom, 2008; Becker y Marecek, 2008) -marco que, como hemos defendido en otros trabajos (Cabanas y Sánchez, 2012), sin embargo comparte con la psicología popular norteamericana en general y con la AA en particular: de ello que el modelo de sujeto que ambos defienden sea tan similar. La AA intentaría también desvincularse de estas mismas raíces pero no apelando a la ciencia, sino al "sentido común", y buscando la complicidad de los lectores cuando dicen describir "las cosas tal y como son" de una forma sencilla y sin complicaciones teóricas -la AA utiliza 1.53\% de terminología de este tipo, muy superior al utilizado tanto por la PP como por la PCC, que utilizan un $0.53 \%$ y un $0.48 \%$, respectivamente.

En cuanto a la categoría retórica de la practicidad, es de esperar que siendo todas ellas tipos de literatura orientadas a facilitar y explicar "estrategias", "guías", "técnicas", "claves", "pasos", etc., utilice en gran medida este tipo de terminología. En la Tabla 3 podemos observar esto, siendo algo más acusado el uso de esta retórica en el caso de la PCC -1.6\%-, que en el de la PP $-1.1 \%$ - y en el de la AA $-1 \%$-, principalmente debido a la enorme frecuencia con que en el primer grupo aparece el términos "técnica" y "técnicas". Podríamos decir que todos estos tipos de literatura están comprometidos con la idea de que la practicidad y la utilidad de sus discursos, guías y técnicas sobre el comportamiento humano conceden un tipo de veracidad empírica a sus propuestas.

\section{Discusión}

La hipótesis principal defendida en este trabajo, a saber, que la frontera psicológica que separa la PP de la AA es, cuanto menos, porosa, queda respaldada con los datos ofrecidos. Por un lado, a nivel psicológico y conceptual subyace un modelo de sujeto muy similar en ambos tipos de literatura, es decir, un conjunto de conceptos y de caracterizaciones psicológicas muy semejantes para hablar, describir y explicar el comportamiento de los individuos. Por otro lado, las distinciones entre la PP y la AA tienden a guardar más relación con aspectos superficiales, como el tipo de retórica que utilizan, que con aspectos teóricos, conceptuales y psicológicos más profundos. Ambos tipos de literatura 1) fomentan un modelo de sujeto que ya define un conjunto y no otro de preferencias e intereses aceptables y deseables, privilegiando aquellos que benefician personalmente al individuo; 2) teorizan que la felicidad es un objetivo natural y un estado que se consigue mediante el pleno control, gestión y conocimiento del "interior" de cada cual, que nos rodean; 3 ) defienden que nos responsabilicemos por completo de nuestros éxitos y de nuestros fracasos, y hacen especial hincapié en la necesidad de que nos controlemos, nos vigilemos y nos censuremos constantemente en nombre de su propuesta sobre la felicidad; 4) también promueven que pongamos especial énfasis en la constante mejora de nuestras capacidades, en el incremento de nuestro capital humano y en la consecución de nuestros propios objetivos. Tienen, por tanto, implicaciones y aplicaciones psicológicas muy similares, y su separación institucional no nos debería hacer pasar por alto las comunalidades históricas, culturales, sociales y psicológicas que existen entre ambas.

Los resultados obtenidos respaldan también cada una de las hipótesis secundarias, pues como hemos visto, las categorías psicológicas del individualismo "positivo" no sólo están fuertemente presentes tanto en la PP como en la AA, sino que también son igualmente relevantes para ambos tipos de literatura. Además, aunque tales categorías están presentes también en corrientes psicológicas como la cognitivoconductual, ni son tan importantes -ni por separado, ni en conjunto- para la misma, ni se conceptualizan de la misma forma, indicando que muchas de las semejanzas tienden a ser más una cuestión de significante que de significado.

Si bien son mucho mayores los elementos comunes que PP y AA comparten, también hemos señalado ciertas diferencias entre ambos tipos de literatura en torno a estas mismas categorías, destacando el tipo de terminología preferente que caracteriza a cada una de ellas. Así, la PP hace un uso destacado de la terminología del bienestar y de las actitudes positivas, así como de la mejora y el crecimiento personales -florecimiento-, conceptos que conforman su seña de identidad particular y que juegan un papel principal en la definición de lo que ellos defienden como su objeto de estudio. Por su parte, la AA enfatiza el autocontrol y el cambio como elementos principales sobre los cuales desarrollar su discurso sobre la naturaleza del individuo, además de presentar mayor cantidad de terminología genérica del interior para definir el espacio psíquico de los sujetos.

También hemos analizado el uso retórico que cada tipo de literatura pone en juego a la hora de defender sus argumentos. Según los resultados, los tres tipos de literatura esgrimen la practicidad y utilidad como argumentos a favor de la defensa de sus conceptos y de las tecnologías psicológicas que ofrecen, algo que destacaba especialmente en la PCC por su explicitud en la presentación de técnicas de modificación de conducta. La PCC destacaba también por el marcado uso de un vocabulario clínico propio y su apoyo en terminología médica también especializada. La AA, por su parte, a diferencia de la PCC y la PP esgrimía explicaciones "autoevidentes" de tipo universalista para apoyar sus argumen- 
tos. En el caso de la PP, compartía con la PCC el recurso terminología de tipo metodológico, pero a diferencia de la segunda la primera destacaba por hacer uso profuso de terminología de tipo científico. A este respecto comentamos que el uso de este tipo de retórica respondía a dos objetivo principales: la demarcación disciplinar respecto de la AA y la ganancia en legitimidad académica y en poder persuasivo.

\section{Conclusiones}

En conclusión, existen semejanzas y diferencias entre todos los tipos de literatura analizados, seguramente más de unas y de otras de las que aquí se han señalado. Así y todo, los análisis presentados en este trabajo sugieren una notable semejanza entre la PP y la AA en torno al modelo de sujeto aquí presentado, modelo que, por su parte, no caracterizan otras propuestas psicológicas con similares objetivos interventivos y aplicados como la PCC. A nuestro modo de ver, las semejanzas entre AA y PP se deben a que ambos son herederos de un conjunto de prejuicios, de expectativas y de demandas

\section{Referencias}

Baccalá, de la Cruz, y Scheuer, N. (2002). Una Aplicación de la Lexicometría a la Descripción de Procesos Evolutivos en Psicología. JADT. Ges Journées internationales d'Analyse statistique des Données Textuelles.

Baptista, J. M., y Sureda, J. (1987). Análisis de Correspondencias y Técnicas de Clasificación. Infancia y Aprendizaje, 39-40. 171-186.

Bautista, A., Pérez Echeverría, M. P., Pozo, I., De la Cruz, M., y Scheuer, N. (2006). La Lexicomtería Aplicada al Estudio de las Concepciones sobre la Enseñanza y el Aprendizaje. Concepciones de Profesores de Piano con Distinta Experiencia Docente. JADT. 8es Journées internationales d'Analyse statistique des Données Textuelles.

Becker, D. y Macerek, J. (2008). Positive Psychology: History in the Remaking? Theory and Psychology, 18 (5), 591-604.

Binkley, S. (2011). Situating Psychological Well-Being: Exploring the Cultural Roots of Its Theory and Research. Subjectivity, 4 (4), 371-394.

Biswas-Diener, R., y Dean, B. (2007). Positive Psychology Coaching. Putting the Science of Happiness to Work for Your Clients. New Yersey: John Wiley \& Sons, Inc.

Boltansky, L., y Chiapello, E. (2007). The New Spirit of Capitalism. New York: Verso.

Cabanas, E. (2011a). Revisión de los libros Ehrenreich, B. (2009). Smile or Die: How positive thinking fooled America and the world. London: Granta Books; y Vázquez, C. y Hervás, G. [coords.] (2009). La ciencia del bienestar: fundamentos de una Psicología Positiva. Madrid: Alianza Editorial. Estudios de Psicología, 32 (2), 278-284.

Cabanas, E. (2011b). El Individualismo "Positivo" y sus Categorias Psicológicas: una Introducción al Estudio de la Historiogénesis y de las Consecuencias Psicologicas, Politicas y Económicas de la Psicología Positiva. Proyecto para la obtención del DEA. UAM: Departamento de Psicología Básica.

Cabanas, E., y Sánchez, J. C. (2012). Las Raíces de la Psicología Positiva. Papeles del Psicólogo, 33 (3), 172-182.

Catellví, M. T. (1978). La Lexicometría como Método de Localización de Rasgos Ideológicos. Revista española de lingüistica, 2. 335-344.

Christopher, J. (1999). Situating Psychological Well-Being: Exploring the Cultural Roots of Its Theory and Research. Journal of Counseling \& Development, 77, 141-152.

Christopher, J. y Hickimbottom, S. (2008). Positive Psychology, Ethnocentrism, and the Disguised Ideology of Individualism. Theory and Psychology, 18 (5), 563-589.

Ehrenreich, B. (2009). Smile or Die: How positive thinking fooled America and the world. London: Granta Books. sobre el comportamiento de los individuos que está enormemente enraizado y presente en el conocimiento popular norteamericano.

No obstante, son necesarios más estudios al respecto, tanto históricos y teóricos como empíricos. A este este respecto, y como hemos destacado desde el comienzo, la fuerza del análisis lexicométrico y del análisis factorial de correspondencias viene más determinada más por la calidad de la justificación teórica y por la plausibilidad del análisis y la interpretación de los datos que por su valor como técnica estadística y analítica en sí misma -algo que, por otro lado, tiende a ocurrir con toda técnica de análisis estadístico. Es además conveniente que este tipo de metodología venga complementada con otra de tipo más cualitativo. Por nuestra parte, aunque en este trabajo hemos utilizado la técnica KWIC para contextualizar varios de los términos y aportar ejemplos de cada una de las categorías, serían necesarios estudios que utilizaran a fondo este tipo de metodología cualitativa.

Fernández-Ríos, L., y Novo, M. (2012). Positive Pychology: Zeigeist (or spirit of the times) or ignorance (or disinformation) of history? International Journal of Clinical and Health Psychology, 12 (2), 333-344.

Feher, M. (2009). Self-Appreciation; or, The Aspirations of Human Capital. Public Culture, 21 (1)

Greenacre, M. (2008). La Práctica del Análisis de Correspondencias. España: Rubes Editorial.

Held, B. (2001). Stop Similing, Start Kvetching: A 5-Step Guide to Creative Complaining. New York: St. Martin's Griffin.

Held, B. (2004). The Negative Side of Positive Psychology. Journal of Humanistic Psychology, 44 (1), 9-46.

Illouz, E. (2010). La Salvación del Alma Moderna. Terapia, Emociones y la Cultura de la Autoayuda. Buenos Aires: Katz Ediciones.

Íñiguez, L. (2006). Análisis del Discurso: Manual para las Ciencias Sociales. Barcelona: Editorial UOC.

Johnson, R., y Onwuegbuzie, A. (2007). Toward a Definition of Mixed Method Research. Journal of Mixed Methods Research, 1 (2), 112-133.

Landauer, T. K., y Dumais, S. T. (1997). A Solution to Plato's Problem: The Latent Semantic Analysis Theory of Acquisition, Induction, and Representation of Knowledge. Psychological Review, 104 (2), 211-240.

Lazarus, R. S. (2003). Does the Positive Psychology Movement Have Legs? Psychological Inquiry, 14 (2), 93-109.

Leech, N., y Onwuegbuzie, A. (2008). Qualitative Data Analysis: A Compendium of Techniques and a Framework for Selection for School Psychology Research and Beyond. School Psychology Quarterly, 23 (4), 587604.

Lebart, L., Salem, A., y Bécue, M. (2000). Análisis Estadístico de Textos. Lleida: Editorial Milenio.

Lipovetsky, G. (2007). La Felicidad Paradójica. Ensayo sobre la Sociedad de Hiperconsumo. Barcelona: Editorial Anagrama.

Linley, P., y Joseph, S. (2004). Positive psychology in practice. John Wiley \& Sons, Inc., Hoboken, New Jersey.

Lyubomisrsky, S. (2007). The How of Happiness: A New Approach to Getting the Life you Want. New York: The Penguin Press.

Lyubomirsky, S., Shledon, K. M., y Schkade, D. (2005). Pursuing happiness: The architecture of sustainable change. Review of General Psychology, 9 (2), 111-131.

Miller, A. (2008). A Critique of Positive Psychology -or 'The New Science of Happiness'. Journal of Philosophy of Education, 42 (3-4), 591-608. 
Norem, J. (2001). El Poder Positivo del Pensamiento Negativo. Utiliza el Pensamiento Negativo para Reducir tu Ansiedad y Rendir al Máximo. Editorial Paidós. Barcelona.

Papalini, V. A. (2010). Libros de Autoayuda: Biblioterapia para la Felicidad. Athenea Digital, 19.

Parker, I. (2010). La Psicología como Ideología. Contra la Disciplina. Madrid: Catarata.

Peterson, C., y Seligman, M. (2004). Character Strengths and Virtues: A Handbook and Classification. New York: Oxford University Press.

Pérez-Álvarez, M. (2012). La Psicología Positiva: Magia Simpática. Papeles del Psicólogo, 33 (3), 183-201.

Rimke, H. (1997). (Re)Constructing the Ethical Self: Self-Help Literature as a Contemporary Project of Moral Regulation. Thesis. Ottawa: Carleton University.

Rimke, M. (2000). Governing Citizens Through Self-help Literature. Cultural Studies, 14 (1), 61-78.

Romeu, L. (1991). Análisis Factorial de Correspondencias: Aplicación al Vocabulario de una Muestra de Prensa (1939/45). Revista de Filologia, 7, 615-632.
Rose, N. (1998). Inventing our Selves: Psychology, Power and Personhood. London: Cambridge university press.

Ryan, G., y Bernard, H. (2003). Techniques to Identify Themes. Field Methods, 15 (1) 85-109.

Seligman, M. (2005). La Auténtica Felicidad. Barcelona: Byblos.

Seligman, M. (2011). La Vida que Florece. Barcelona: Ediciones B.

Seligman, M., y Csikszentmihalyi, M. (2000). Positive Psychology. An Introduction. American Psychologist, 55 (1), 5-14.

Vázquez, C., y Hervás, G. [Eds.] (2008). Psicología positiva aplicada. Bilbao: Biblioteca de Psicología. Desclée de Brouwer.

Vázquez, C. y Hervás, G. [Eds.] (2009). La Ciencia del Bienestar: Fundamentos de una Psicología Positiva. Madrid: Alianza Editorial.

Vera Poseck, B. (2006). Psicología Positiva: Una nueva forma de entender la Psicología. Papeles del Psicólogo, 17, 3-8.

Wetherell, M. (1998). Positioning and interpretative repertoires: Conversation analysis and poststructuralism in dialogue. Discourse and Society, 9 (3), $387-412$.

(Artículo recibido: 20-2-2013; revisión recibida: 11-9-2013; aceptado: 22-1-2014) 